\title{
The relative importance of host characteristics and co-infection in generating variation in Heligmosomoides polygyrus fecundity
}

\author{
L. T. LUONG ${ }^{1 *}$, S. E. PERKINS ${ }^{1}$, D. A. GREAR ${ }^{1}$, A. RIZZOLI ${ }^{2}$ and P. J. HUDSON ${ }^{1}$ \\ ${ }^{1}$ Center for Infectious Disease Dynamics, Penn State University, University Park, PA 16802 USA \\ ${ }^{2}$ Edmund Mach Foundation, IASMA Research and Innovation Centre, S. Michele all'Adige, Trento, Trentino, Italy
}

(Received 19 October 2009; revised 3 November and 13 November 2009; accepted 18 November 2009; first published online 29 Fanuary 2010)

S UMMAR Y

We examined the relative importance of intrinsic host factors and microparasite co-infection in generating variation in Heligmosomoides polygyrus fecundity, a parameter that serves as a proxy for infectiousness. We undertook extensive trapping of Apodemus flavicollis, the yellow-necked mouse in the woodlands of the Italian Alps and recorded eggs in utero from the dominant nematode species H. polygyrus, and tested for the presence of five microparasite infections. The results showed that sex and breeding status interact, such that males in breeding condition harboured more fecund nematodes than other hosts; in particular, worms from breeding males had, on average, $52 \%$ more eggs in utero than worms from nonbreeding males. In contrast, we found a weak relationship between intensity and body mass, and no relationship between intensity and sex or intensity and breeding condition. We did not find any evidence to support the hypothesis that co-infection with microparasites contributed to variation in worm fecundity in this system. The age-intensity profiles for mice singly-infected with $H$. polygyrus and those co-infected with the nematode and at least one microparasite were both convex and not statistically different from each other. We concluded that intrinsic differences between hosts, specifically with regard to sex and breeding condition, contribute relatively more to the variation in worm fecundity than parasite co-infection status.

Key words: super-shedders, male-biased transmission, Heligmosomoides polygyrus, Apodemus, parasite community, co-infection.

\section{INTRODUCTION}

Empirical studies have identified that individual hosts vary greatly in terms of both the rate at which they produce infectious particles and the rate at which infectious individuals come into contact with susceptible individuals. In terms of potential onward transmission, individuals can be classified as the 'insignificant many' or the 'vital few' (Woolhouse et al. 1997; Perkins et al. 2003; Ferrari et al. 2004). Amongst macroparasite infections, the majority of a helminth population is usually concentrated in a minority of the host population, and is invariably best described by the negative binomial distribution in parasite intensity, because the variance in the intensity of infection between populations rises faster than the mean (e.g. Shaw and Dobson, 1995; Wilson et al. 2001). There are 3 ways the vital few individuals may be responsible for higher than average number of transmission events: (1) these individuals have

* Corresponding author: Center for Infectious Disease Dynamics, Penn State University, University Park, PA 16802 USA. Tel: + 814865 0522. Fax: +8148659131. E-mail: 1t11@psu.edu higher than average contact rates with susceptible hosts, irrespective of their intensity of infection; (2) these individuals produce infectious particles that survive longer or are more infectious than others; (3) these hosts shed more infectious particles than others in the population (Anderson and May, 1991; Matthews et al. 2006; Chase-Topping et al. 2008). Heterogeneity in any one or combination of these processes can result in an increase in the basic reproductive number $\left(\mathrm{R}_{\mathbf{0}}\right)$ and hence the spread of infection (Woolhouse et al. 1991, 1997; Smith et al. 2007).

Our study pertains specifically to the third mechanism, with the aim to identify the relative contribution of host characteristics and parasite co-infection status in generating variation in fecundity of the nematode $H$. polygyrus. Variation in egg output per infected host need not be linearly related to variation in the nematode intensity, particularly if worm growth and fecundity are constrained by density dependence via intra-specific competition or immune-mediated host responses, and perhaps exacerbated by co-infection (Goater, 1992; Tompkins and Hudson, 1999; Dezfuli et al. 2001 ; Paterson and Barber, 2007). Hence, we used in utero egg count as 
an estimate of nematode fecundity (Ractliffe and LeJambre, 1971; McClelland, 1980; Smith, 1988; Szalai and Dick, 1989; Marcogliese, 1997; Tompkins and Hudson, 1999). This parameter is arguably more reliable than the alternative approach of counting worm eggs expelled by the host in the faeces, owing to the variability in faecal egg counts that can occur within and between days (Anderson and Schad, 1985; Keymer and Slater, 1987; Stear et al. 1995; Harvey et al. 1999). As such, eggs per gram (EPG) data from a few time-points may not accurately reflect a host's infectiousness. Assuming that the rate of egg production (i.e., the nematode's lifetime reproductive or shedding rate) is linearly related to the number of eggs in the nematode uterus, the number of eggs in utero serves as a reliable estimate of the number of eggs shed into the environment (Michel, 1963 ; Ractliffe and LeJambre, 1971). Previous work has shown this to be indeed the case in nematodes, where a positive association was found between the number of eggs in utero and the faecal egg count in sheep infected with Teladorsargia circumcinta (Stear et al. 1995 ; Stear and Bishop, 1999). Here, we assume that in utero egg count is a reliable measure of worm fecundity in $H$. polygyrus, which serves as a proxy for host infectiousness.

Hosts harbouring highly fecund worms are likely to shed more infectious particles than others in the population, and hence contribute more to transmission events than the average host. Studies on H. polygyrus but also on other nematode and tick vectors have demonstrated that male hosts, particularly those in breeding condition may be responsible for the majority of parasite transmission (Perkins et al. 2003; Ferrari et al. 2004; Luong et al. 2009). Furthermore, variation in the intensity of nematode infection between individuals has been related to host body mass and sex (Viney, 2002; Paterson and Viney, 2002; see review by Poulin, 1996) and will also have a major influence on variation in individual host infectiousness. In this study, we examined the relationship between host characteristics and nematode fecundity by quantifying the number of in utero eggs of individual worms in wild mice. We asked, 'Do inherent variations among individual hosts contribute to the variation in nematode fecundity and hence production of parasite transmission stages'? We hypothesized that intrinsic differences between individual hosts with regard to sex, breeding status and body mass generate variation in nematode fecundity. Since males generally harbour a higher intensity of parasites, we expected that breeding males harboured nematodes with higher fecundity than other hosts. As a contrast to the relationship between worm fecundity and host characteristics, we also examined the relationship between worm intensity and host characteristics.

Co-infecting parasites and pathogens have also been proposed to affect the number of infective stages produced (Bassetti et al. 2005; Matthews et al. 2006). The hypothesis here is that individuals that carry co-infections are more susceptible than single infected hosts, and that these nematodes are also more fecund. For example, co-infection with the poxyvirus myxoma has been shown to be positively associated with increased susceptibility to the nematode, Trichostronglyus retortaeformis (Cattadori et al. 2007). This interaction is thought to be often mediated by the host immune system whereby pathogens exert a suppressive effect on the cytokine response, stemming the control of nematode establishment, expulsion and fecundity (Cox, 2001; Lello et al. 2004; Graham, 2008; Montes et al. 2009). Thus, we asked 'How do inter-specific interactions between the parasite community influence nematode fecundity and intensity'? We proposed that coinfecting microparasites indirectly generate variation in nematode fecundity such that worms from hosts not previously exposed to microparasites are expected to have fewer eggs in utero than worms collected from hosts that were exposed.

\section{MATERIALS AND METHODS}

\section{Animal trapping and pathogen sampling}

Extensive trapping of Apodemus flavicollis, the yellow-necked mouse was carried out in July 2002 using multi-capture live traps (Ugglan type 2, Graham Sweden) at 6 study sites for 3 nights, with a total trapping effort of 3456 trap nights. The study sites were located in mixed broadleaf woodlands of the Italian Dolomitic Alps. The methods followed standard trapping procedures and are described elsewhere (Ferrari et al. 2007). For each mouse we recorded the host sex, body mass and breeding condition. Individuals with descended testes, perforated vagina or pregnancy were recorded as animals in breeding condition. Mice with a body mass above $15 \mathrm{~g}$ were euthanized following the animal procedures of the European Commission Directive 86/ 609/EEC implemented by Italy. The gastro-intestinal tract was removed and all helminths extracted using a filtration-sedimentation technique (Euzeby, 1982). All helminths were counted and identified to species level. The dominant helminth species was Heligmosomoides polygyrus, a directly transmitted nematode common in A. flavicollis (Gregory et al. 1992; Ferrari et al. 2004). From the larger dataset we randomly selected a subset $(25 \%, n=59$ mice $)$ to carry out in utero egg counts. From these mice each female $H$. polygyrus was mounted onto an individual slide and the eggs in utero were visually counted under a stereomicroscope. A subsample of nematodes $(n=24)$ from this group was measured to assess the relationship between worm length and in utero egg count. Individual $H$. polygyrus were photographed under a stereomicroscope (Leica $\mathbb{R}$ S6E) 
with a digital camera (Nikon ( Coolpix 4500). The length of each nematode was measured using the ImageJ software package (Rasband, 2007).

Blood and tissue samples were taken from each individual to test if the mice had been exposed to the following pathogens: arenavirus, cowpox, Ehrlichia spp., murine-herpes gammavirus (MuHV), and tickborne encephalitis (TBE). Serum samples were analysed using standard enzyme-linked immunosorbent assay (ELISA) and haemaglutination inhibition (HI) assay techniques for TBE as described in Perkins et al. (2006) and Rizzoli et al. (2007). Serum samples were tested for the arenavirus and MuHV using an indirect immunofluorescent (IF) antibody assay as described in Tagliapietra et al. (2009) and Telfer et al. (2007), respectively. Cowpox virus antibody was also determined in an IF antibody assay as described in Crouch et al. (1995). Standard PCR and DNA sequencing were used for the identification of Ehrlichia from liver and spleen samples taken at necropsy (Liz et al. 2000). We recognize that animals that were seropositive for a given pathogen may not have been actively infected with the microparasite at the time of necropsy (see Discussion section).

\section{Host characteristics and $\mathrm{H}$. polygyrus intensity}

The relationship between nematode intensity and host factors was analysed with a generalized linear model using a negative binomial error distribution. Nematode intensity per host was modelled as the response with host mass, sex, breeding condition, coinfection of any microparasite, and their interactions as explanatory variables. We used backwards model selection starting with the full model with all interaction terms to determine a minimum best-fit model. We removed explanatory terms if a drop-in-deviance $\chi^{2}$ test comparing a full model to a reduced model was not significant $(P>0 \cdot 05)$. We first tested the most complex interaction terms and proceeded with less complex interaction terms until we reached a minimum model.

\section{Host characteristics and $\mathrm{H}$. polygyrus fecundity}

To investigate the relationship between host characteristics and nematode fecundity, we used the logarithm of in utero egg counts of female $H$. polygyrus from a subset ( $n=59$ hosts) of the full data as the response variable in a linear mixed effect (LME) regression model. Host body mass, host sex, and host breeding condition were entered into the model as fixed-effect explanatory variables. To account for the replicated effect of measuring multiple worms from the same host, the unique identification code of each host was included as a random effect. We again used backwards model selection starting with the full model with all interaction terms to determine a minimum best-fit model. A posteriori contrasts were employed to test for differences in mean nematode fecundity between specific functional host-groups, which were defined as non-breeding females, breeding females, non-breeding males, and breeding males.

Age and co-infection relationship with $\mathrm{H}$. polygyrus intensity

Body mass was used to estimate the age class of each non-juvenile $(\geqslant 19 \mathrm{~g}$; Bajer et al. 2005; where $19 \mathrm{~g}$ was the smallest animal recorded in breeding condition). We created 6 equal-sized age classes with increments of $3.85 \mathrm{~g}$. The relationship between age class, $H$. polygyrus intensity and co-infection was investigated by fitting the observed data to hypothetical age-intensity curves and determining the best fit. The fits investigated included linear and second order polynomial, which were chosen according to previously described age-intensity distributions (Wilson et al. 2001). Models were built using pathogen co-infection as an additive factor and as an interaction factor between the linear and polynomial age variables; giving 8 competing models to compare (Table 3). We conducted a Pearson $\chi^{2}$ goodness-offit test on the full model. Model comparisons were carried out using Akaike's information criterion (AIC) value and relative importance of variables in the candidate models was evaluated by summing the model weight $\left(\omega_{\mathrm{i}}\right)$ of the models containing a parameter of interest (Burnham and Anderson, 2002). The individual $\left(\omega_{\mathrm{i}}\right)$ indicates the probability that a given model is the best model of the candidate set given the data, the cumulative $\left(\omega_{\mathrm{i}}\right)$ refers to the probability that a subset of models contains the best fit model among competing models, and variable weight is an index of the relative importance of an individual explanatory variable among the candidate set of models.

\section{RESULTS}

\section{Parasite community}

We captured a total of $230 \mathrm{~A}$. flavicollis, from which 6 species of nematode parasites were recovered (Table 1). The directly transmitted nematode $H$. polygyrus was most prevalent at $66 \%$ and also reached the highest mean intensity at $7 \cdot 02 \pm 0 \cdot 80$ (Table 1). This intensity distribution followed a negative binomial distribution (goodness-offit test $P>0 \cdot 1)$ with mean intensity $=7 \cdot 02(95 \%$ CI $[5 \cdot 70,8 \cdot 84]$ and with an estimated dispersion parameter from the negative binomial, $k=0 \cdot 34(95 \%$ CI $[0 \cdot 30,0 \cdot 44]) . H$. polygyrus body length was correlated with the number of eggs in utero per female worm $($ Coef. $=0 \cdot 107$, s.E. $=0 \cdot 034, P=0 \cdot 002)$. The 
Table 1. Summary of the prevalence and intensity ( \pm s.e.) of infection for six species of parasitic nematodes recovered from the wild rodent, Apodemus flavicollis

(Co-infection indicates the percentage of the population which is simultaneously co-infected with Helgimosomoides polygyrus and another given species of gastrointestinal parasitic nematode.)

\begin{tabular}{llll}
\hline \hline Species & $\begin{array}{l}\text { Prevalence } \\
(\%)\end{array}$ & Intensity & $\begin{array}{l}\text { Percentage } \\
\text { co-infection }\end{array}$ \\
\hline H. polygyrus & $65.7 \pm 3.1$ & $7.02 \pm 0.80$ & NA \\
Syphacia frederici & $10.7 \pm 1.9$ & $2.18 \pm 0.32$ & 20.9 \\
Syphacia spp. & $9.9 \pm 1.9$ & $0.74 \pm 0.23$ & 4.78 \\
Trichuris muris & $3.5 \pm 1.2$ & $0.04 \pm .012$ & 2.17 \\
Syphacia stroma & $18.3 \pm 2.4$ & $0.10 \pm 0.05$ & 1.30 \\
Rictularia proni & $8.7 \pm 1.8$ & $0.04 \pm 0.02$ & 0.43 \\
\hline \hline
\end{tabular}

Table 2. Summary of the prevalence of antibodies to six different pathogens from a free-living population of Apodemus flavicollis

(The percentage co-infection indicates the frequency at which the given pathogen co-occurs with Heligmosomoides polygyrus.)

\begin{tabular}{lrl}
\hline \hline Microparasite & Prevalence & $\begin{array}{l}\text { Percentage } \\
\text { co-infection }\end{array}$ \\
\hline MuHV & $24.55 \pm 0.03$ & 18.64 \\
Lyme & $17.90 \pm 0.03$ & 11.79 \\
Ehrlichia & $12.61 \pm 0.02$ & 9.57 \\
Arenavirus & $10.22 \pm 0.02$ & 4.44 \\
Cowpox & $2.71 \pm 0.01$ & 1.81 \\
TBE & $3.59 \pm 0.01$ & 1.35 \\
Any pathogen & $56.02 \pm 0.04$ & 35.65 \\
\hline \hline
\end{tabular}

most common nematode co-infection occurred with $H$. polygyrus and Syphacia frederici at $20.9 \%$ (Table 1).

Sera from mice were analysed for antibodies to 5 different pathogens. Of the pathogens tested, mice had the highest antibody-positive sero-prevalence against murine herpes gammavirus (MUHV) $(24 \cdot 5 \pm$ $0.03 \%)$, which also had the highest percentage of coinfection $(18 \cdot 64 \%$ ) with $H$. polygyrus (Table 2$)$. The majority of animals $(56 \cdot 0 \pm 0 \cdot 04 \%)$ had antibodies against at least 1 of the pathogens screened. Co-infection with $H$. polygyrus and any given pathogen occurred at a prevalence of $35 \cdot 6 \%$ (Table 2 ).

\section{Host characteristics and $\mathrm{H}$. polygyrus intensity}

A generalized linear model (GLM) analysis of the intensity of $H$. polygyrus and host factors identified a weak but significant positive association between the intensity of infection and host body mass (Coef. $=0 \cdot 041$, s.E. $=0 \cdot 017, P=0 \cdot 019$ ). The intensity of infection (uninfected mice included) was significantly (Coef. $=-1 \cdot 85$, s.E. $=0 \cdot 446, P<0 \cdot 001$ ) lower among mice in breeding condition (mean \pm
s.E. $=3 \cdot 74 \pm 1 \cdot 16)$ than those not in breeding condition $($ mean \pm s.e. $=8 \cdot 88 \pm 0 \cdot 75)$. Host sex $($ Coef. $=$ $0 \cdot 037$, s.e. $=0.272, P=0 \cdot 891)$ and the interaction with breeding status and sex were not significant (Coef. $=0 \cdot 832$, s.E. $=0 \cdot 522, P=0 \cdot 111$ ). The relationship between $H$. polygyrus intensity and $S$. frederici intensity was not significant (Coef. $=0 \cdot 009$, s.E. $=$ $0 \cdot 028, P=0 \cdot 754)$. There were no statistically significant differences in the intensity of $H$. polygyrus in relation to the presence or absence of microparasite antibodies (Coef. $=0 \cdot 149$, s.E. $=0 \cdot 237, P=0.53$ ).

\section{Host characteristics and $\mathrm{H}$. polygyrus fecundity}

We measured in utero egg counts from a total of 59 A. flavicollis hosting a total of 652 nematodes, from which 417 intact female worms were sampled. The mean number of eggs in utero was not correlated with the intensity of $H$. polygyrus (Coef. $=-0 \cdot 681$, S.E. $=0 \cdot 420, P=0 \cdot 112)$. A linear-mixed-effects regression analysis of host factors revealed that host body mass was significantly negatively associated with the number of eggs per worm (Coef. $=-0 \cdot 039$, D.F. $=327, P<0.0001$; Fig. 1). Host sex (Coef.= $0 \cdot 084$, s.E. $=0 \cdot 065, P=0 \cdot 203)$ or breeding status alone was not significant (Coef. $=-0 \cdot 156$, s.E. $=$ $0 \cdot 151, P=0 \cdot 308)$, but the interaction between sex and breeding status was significantly associated with nematode fecundity (Coef. $=0.521$, D.F. $=53$, $P=0 \cdot 004)$. Co-infection with another dominant nematode parasite, $S$. frederici did not influence $H$. polygyrus fecundity (Coef. $=0 \cdot 005$, s.E. $=0 \cdot 011$, $P=0.681$ ). The presence or absence of antibodies to any pathogen did not significantly alter $H$. polygyrus fecundity (Coef. $=0 \cdot 125$, s.E. $=0 \cdot 076, P=0 \cdot 10$ ).

To further examine the interaction between sex and breeding condition, an a posteriori contrast analysis on the functional groups was conducted. On average $H$. polygyrus that infected breeding males were $52 \%$ more fecund than those infecting nonbreeding males (Coef. est. $=0 \cdot 411$, s.E. $=0 \cdot 148$, $P=0 \cdot 006)$. Worms from breeding males were also 


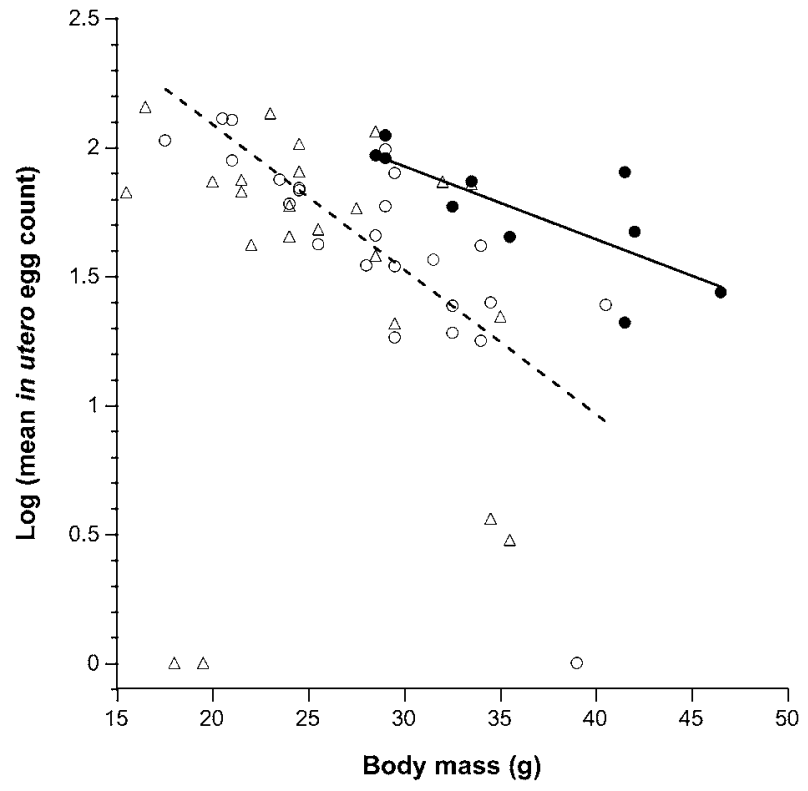

Fig. 1. The relationship between host body mass of wild rodents and the nematode fecundity. Breeding (solid circles) and non-breeding (open circles) males have a negative relationship between worm fecundity and body mass (black line, breeding males; dashed line, non-breeding males). Note that for a given mass, breeding males harboured more fecund nematodes on average compared to non-breeding males. The association between egg count and body mass was not significant for non-breeding females (open triangle); 2 females, both pregnant have been omitted. The 3 individuals with no eggs are likely to be immature nematodes.

more fecund on average than those infecting nonbreeding females (Coef. $=-0 \cdot 567$, s.E. $=0 \cdot 194, P=$ $0 \cdot 003)$. The mean in utero egg count for worms from non-breeding males exhibited a tendency to be higher than for non-breeding females, but this was not statistically significant (Coef. $=0 \cdot 256$, s.E. $=0 \cdot 136$, $P=0 \cdot 060$ ). Worm fecundity was not significantly different for breeding and non-breeding female mice (Coef. $=-0 \cdot 193$, s.E. $=0 \cdot 273, P=0 \cdot 479)$. The sample size of breeding females was low $(n=2)$ and therefore lacked sufficient statistical power to detect differences among groups.

\section{Age and co-infection relationship with $\mathrm{H}$. polygyrus intensity}

Next, we tested the hypothesis that co-infection with a microparasite would influence the intensity of $H$. polygyrus infection. Out of the 8 competing models, the top 4 candidate models explained a cumulative $94 \%$ of the model weights indicating that there is a high probability that one of the top 4 models is the best model of the candidate models (Table 3). A Pearson goodness of fit test showed that the full model was an adequate fit to the data $(P=0 \cdot 84)$. However, no single model had strong support as the best model. We calculated the variable weights in order to assess which explanatory variables contained in the top 4 models were important predictors of $H$. polygyrus intensity and the linear predictor of age, as well as the polynomial predictor of age was important in our candidate set of models ( 0.94 and 0.79 variable weights, respectively). Variable weights of the interaction terms between age (linear component) and pathogen co-infection, as well as age (polynomial component) and pathogen co-infection were low $(0 \cdot 11$ and $0 \cdot 12$, respectively). Hence, nematode intensity increased with host age, peaked in the middle age classes before decreasing in older animals. Although the additive effect of pathogen co-infection was an important component of the candidate models (variable weight 0.98 ), the age-intensity profile did not differ greatly between mice singly-infected with $H$. polygyrus and mice coinfected with $H$. polygyrus and a pathogen (Fig. 2).

\section{DISCUSSION}

We examined the relative importance of intrinsic host factors and microparasite co-infection in generating variation in $H$. polygyrus fecundity, a proxy for infectiousness. Studies on other parasite-host systems have suggested that a small proportion of the population is responsible for the majority of transmission events (Woolhouse et al. 1997; Perkins et al. 2003; Galvani and May, 2005). There are several possible mechanisms underlying this heterogeneity in transmission, including variation in contact rates between infected and susceptible hosts, and the quality and/or quantity of infective stages shed into the environment (Keeling et al. 2001; Matthews et al. 2006; Chase-Topping et al. 2008). In our study, we investigated the relative contribution of intrinsic differences between hosts and co-infection status to variation in the quantity of infective stages shed, measured here in terms of nematode fecundity. Our results show that sex and breeding status interacted such that breeding males harboured more fecund nematodes than other hosts. In contrast, we did not find any evidence to support the hypothesis that co-infecting pathogens generated variation in worm fecundity. The presence or absence of antibodies to any pathogen did not significantly affect worm fecundity, and the age-intensity profiles for these two groups did not differ significantly from each other. Taken together, these results suggest that intrinsic differences between hosts, specifically with regard to sex and breeding condition, contribute relatively more to the variation in $H$. polygyrus fecundity than co-infection status.

Interestingly, animals of higher body mass harboured nematodes that had a lower fecundity than those of smaller animals. $H$. polygyrus fecundity has been shown to decrease with worm age and eventually cease at $8-10$ weeks post-infection (Keymer and 
Table 3. Candidate models, AIC values, and model weights for 8 generalized linear models testing for linear versus the polynomial fit of the age - Heligmosomoides polygyrus intensity profile, as well as interaction between age-intensity and pathogen co-infection

(The value $\omega_{\mathrm{i}}$ refers to the probability that the model is the best model given the data and the term cumulative $\omega_{\mathrm{i}}$ is the probability that the ascending set of candidate models contains the best model given the data.)

\begin{tabular}{|c|c|c|c|c|c|}
\hline Model & Parameters & AIC & $\triangle \mathrm{AIC}$ & $\omega_{\mathrm{i}}$ & $\begin{array}{l}\text { Cumulative } \\
\omega_{\mathrm{i}}\end{array}$ \\
\hline Age $+(\text { age })^{2}+$ pathogen & 5 & $1137 \cdot 91$ & $0 \cdot 00$ & $0 \cdot 56$ & $0 \cdot 56$ \\
\hline Age + pathogen & 4 & $1139 \cdot 22$ & $1 \cdot 31$ & $0 \cdot 15$ & $0 \cdot 71$ \\
\hline Age + pathogen $+(\text { age })^{2}+(\text { age })^{2} *$ pathogen & 6 & $1139 \cdot 44$ & $1 \cdot 53$ & $0 \cdot 12$ & $0 \cdot 83$ \\
\hline Age + pathogen $+(\text { age })^{2}+$ age $^{*}$ pathogen & 6 & $1139 \cdot 57$ & $1 \cdot 66$ & $0 \cdot 11$ & $0 \cdot 94$ \\
\hline Age + pathogen + age* pathogen & 5 & $1140 \cdot 57$ & $2 \cdot 66$ & $0 \cdot 04$ & $0 \cdot 98$ \\
\hline $\begin{array}{l}\text { Age }+(\text { age })^{2}+\text { pathogen }+ \text { age* pathogen }+ \\
(\text { Age })^{2 *} \text { pathogen }\end{array}$ & $7 *$ & $1141 \cdot 27$ & $3 \cdot 36$ & $0 \cdot 02$ & $>0.99$ \\
\hline Age $+(\text { age })^{2}$ & 4 & $1216 \cdot 46$ & $78 \cdot 55$ & $0 \cdot 00$ & $>0 \cdot 99$ \\
\hline Age & 3 & $1218 \cdot 93$ & $81 \cdot 01$ & $0 \cdot 00$ & $>0 \cdot 99$ \\
\hline
\end{tabular}

* Full model, Pearson goodness-of-fit test $(P=0 \cdot 84)$.

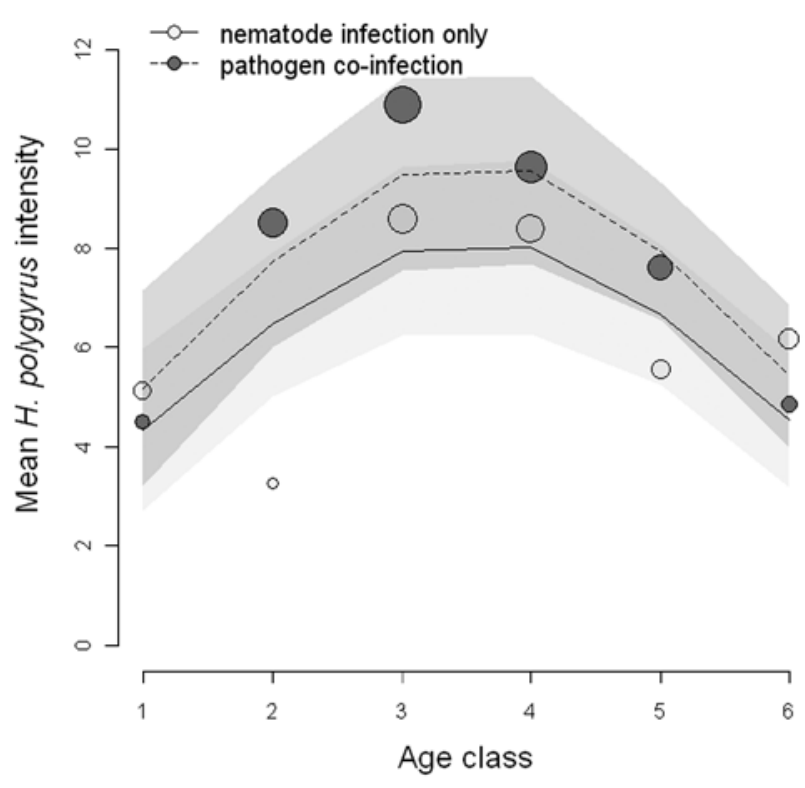

Fig. 2. Age-intensity curves showing the relationship between intensity of Heligmosomoides polygyrus and host age class. The observed mean $H$. polygyrus intensity for mice with the nematode infection only (dark circles) and mice with both nematode and antibodies to pathogen (light grey circles) are shown with size indicating relative sample size. The predicted values for nematode-infected (solid line) and co-infected (dashed line) mice are shown based on a model with the 3 most important relative variable weights, age + age $^{2}+$ pathogen co-infection, with $95 \%$ prediction confidence limits (shaded areas).

Hiorns, 1986; Gregory et al. 1990; Quinnell, 1992). Given that host body mass serves as a proxy for age, the decrease in worm fecundity in larger animals is likely due to the senescence of worms acquired earlier in life. An alternative explanation for the decline in worm fecundity with host age is the development of acquired immunity in older animals (Gregory et al. 1990). Gregory et al. (1992) showed that in repeated nematode infections, nematode egg production decreased over time at a faster rate than in primary infections, suggesting that acquired immunity acts on worm fecundity to constrain parasite population growth. Other model systems such as $T$. retortaeformis have also demonstrated a reduction in worm fecundity with host age as a consequence of acquired immune response (Chylinski et al. 2009).

However, for a given body mass, male hosts in breeding condition harboured significantly more fecund worms than non-breeding males. Possible mechanisms underlying this pattern include hostmediated effects such as immuno-suppression by testosterone, which may be elevated during breeding (Poulin, 1996; Zuk and McKean, 1996). Such immuno-suppression may allow worms to grow larger in the breeding males, thus leading to greater worm fecundity. Indeed, $H$. polygyrus body length was a predictor of the number of eggs in utero in our study. Moreover, Swanson et al. (1984) and Harder et al. (1992) showed that lab mice treated with testosterone implants display a positive relationship between testosterone levels and nematode growth and egg production. An alternative explanation for the higher egg counts among male mice is that breeding males may be subject to greater stress levels as a result of territorial defence and agonistic encounters, compared to non-breeding males (Anderson, 1994; Zuk, 1990). Stress hormones produced by the host under these circumstances including cortisol or corticosterone have been shown to be immunosuppressive (Noble, 1961, 1962, 1966; Stein and Schleifer, 1985; Zuk and McKean, 1996; Elftman et al. 2007).

In contrast to the nematode fecundity results, the intensity of infection was lower among breeding mice compared to non-breeding mice. These results suggest that hosts in breeding condition harbour fewer but more fecund nematodes than non-breeding 
hosts, which is consistent with our findings for male hosts. These contrasting results also illustrate the advantage of using fecundity as a more direct measure of infectiousness than intensity. The general negative relationship between intensity and fecundity may arise as a consequence of intra-specific competition, limiting nematode establishment, growth, or egg production as competition for resources increases. However, the mean number of $H$. polygyrus eggs in utero was not correlated with the intensity of infection, suggesting a lack of density-dependent constraint on per capita female fecundity in this system. Alternatively, a host-mediated mechanism, such as the immune response, may be controlling parasite growth and fecundity (Stear et al. 1995, 1997; Zuk and McKean, 1996; Poulin, 1996). Indeed, Keymer and Hiorns (1986) showed that lab mice subject to repeated infections exhibited an immune-mediated decrease in worm fecundity (also see Gregory et al. 1990). Therefore, breeding animals harbour fewer and more fecund nematodes than non-breeding animals, and this difference can result in higher levels of infectiousness among the mice in breeding condition.

We found no evidence for differences in worm fecundity or intensity of infection between the host sexes. The lack of sex differences conflicts with comparative studies in which male mammals were found to consistently harbour larger and presumably more fecund nematodes (Poulin, 1996; Dare and Forbes, 2008). Both laboratory and field studies of H. polygyrus infections in wood mice (Apodemus sylvaticus) show that males have a higher prevalence and intensity of infection than females (Elton, 1931; O'Sullivan et al. 1984; Gregory et al. 1990; Ferrari et al. 2004). Our inability to detect a significant difference between the sexes may be due to the small sample size among females, particularly those in breeding condition.

The overall age-intensity profile for $H$. polygyrus was convex such that the intensity of $H$. polygyrus peaked in the middle age classes before turning over and declining in the older hosts. This convex shape has also been documented in wild and enclosed populations of $A$. sylvaticus infected with $H$. polygyrus (Gregory, 1991; Gregory et al. 1992). The initial rise in the curve is likely due to an increase in exposure or force of infection with age. Several possible mechanisms may explain the decline in intensity among older age classes, including parasitemediated host mortality, acquired immune response, and/or age-related changes in host exposure to parasite infective stages (Anderson and Gordon, 1982; Anderson and May, 1985; Hudson and Dobson, 1995; Wilson et al. 2001). Parasite-induced host mortality has been demonstrated in lab mice (Keymer and Hiorns, 1986; Scott 1987, 1990) and in enclosed wood mice populations infected with $H$. polygyrus (Gregory, 1991). There is also ample evidence for acquired immunity from a number of laboratory and semi-natural studies of $H$. polygyrus infections in lab Mus (Keymer and Hiorns, 1986; Keymer and Tarlton, 1991; Tanguay and Scott, 1992) and Apodemus species (Gregory et al. 1990; Quinnell, 1992). For example, wood mice infected with $H$. polygyrus exhibited exposure-dependent constraints on parasite population growth, with higher variability in population dynamics between individuals exposed to repeated infections compared to those in primary infection (Gregory et al. 1992; Quinnell, 1992).

Neither the shape nor magnitude of the ageintensity curve was statistically different between single- and co-infected animals. Also, co-infection with at least one microparasite did not influence the intensity or fecundity of $H$. polygyrus compared to hosts infected with the nematode alone. In contrast, laboratory mice experimentally co-infected with $H$. polygyrus and a respiratory bacterium have been shown to be chronic shedders of nematode eggs (Lass et al., personal communication). The failure to detect a difference in our study may be because other naturally occurring pathogens were not considered in the ELISA and HI tests, but may have played a potentially important role in what was categorized as an uninfected animal this study. Furthermore, hosts that tested sero-positive for a pathogen may not be infected at the time of dissection since the ELISA tests merely detected the presence/absence of antibodies, possibly from past infections. If the microparasite had been cleared, then the strength of the pathogen-induced immune response may be diminished, and any immune-mediated interaction between $H$. polygyrus and a microparasite infection could be below the threshold of detection (Janeway and Travers, 1996).

In summary, the present results suggest that intrinsic differences between hosts, specifically the interaction between sex and breeding condition, had a relatively greater impact on nematode fecundity than co-infection with a microparasite. Given that worm fecundity is a proxy for host infectiousness, our results suggest that heterogeneity in the infectiousness of hosts can create super-spreaders, potentially independent of infection intensity. In this case, breeding males were more infectious than other hosts, which supports the findings of Perkins et al. (2003) and Ferrari et al. (2004) that breeding males are driving transmission of several Apodemus parasites. Optimal control of infectious diseases should target treatment of those individuals that account for the majority of the transmission (Anderson and May, 1991; Woolhouse et al. 1997). Future studies that incorporate seasonal data and the experimental manipulation of nematode infections are needed to provide better insight into the role of intrinsic host factors and parasite-community in generating variation in host infectiousness. 
We thank I. Cattadori, A. Pathak, B. Vigliotti for insightful discussions on host immunity, and B. Bozick for assisting with the in utero egg counts. This work was funded by NSF grant EF-0520468 as part of the joint NSF-NIH Ecology of Infectious Disease program, and in part by The Autonomous Provincie of Trento, under grant ECODIS. We also thank the anonymous reviewers for their helpful comments.

\section{REFERENCES}

Anderson, M. (1994). Sexual Selection. Princeton University Press. Princeton, NJ, USA.

Anderson, R. M. and Gordon, D. M. (1982). Processes influencing the distribution of parasite numbers within host populations with special emphasis on parasite-induced host mortalities. Parasitology 85, 373-398.

Anderson, R. M. and May, R. M. (1991). Infectious Diseases of Humans: Dynamics and Control. Oxford University Press, Oxford, UK.

Anderson, R. M. and Schad, G. A. (1985). Hookworm burdens and fecal egg counts: an analysis of the biological basis of variation. Transactions of the Royal Society of Tropical Medicine and Hygiene 79, 812-825.

Bajer, A., Behnke, J., Pawelczyk, A., Kulis, K., Sereda, M. J. and Sinski, E. (2005). Medium-term temporal stability of the helminth component community structure in bank voles (Clethrionomys glareolus) from the Mazury Lake District region of Poland. Parasitology 130, 213-228.

Bassetti, S., Bischoff, W. E. and Sherertz, R. J. (2005). Are SARS superspreaders cloud adults? Emerging Infectious Disease : http://www.cdc.gov/ncidod/EID/ vol11 no04/04-0639.htm.

Burnham, K. P. and Anderson, D. R. (2002). Model Selection and Inference : a Practical InformationTheorectical Approach, 2nd Edn. Springer-Verlag, New York, USA.

Cattadori, I. M., Albert, R. and Boag, B. (2007). Variation in host susceptibility and infectiousness generated by co-infection: the myxoma-Trichostrongylus retortaeformis case in wild rabbits. Fournal of the Royal Society Interface 4, 831-840.

Chase-Topping, M., Gally, D., Low, C., Matthews, L. and Woolhouse, M. (2008). Super-shedding and the link between human infection and livestock carriage of Escherichia coli O157. Nature Reviews Microbiology 6, 904-912.

Chylinski, C., Boag, B., Stear, M. J. and Cattadori, I. M. (2009). Effects of host characteristics and parasite intensity on growth and fecundity of Trichostrongylus retortaeformis infections in rabbits. Parasitology 136, 117-123.

Cox, F. E. G. (2001). Concomitant infections, parasites and immune responses. Parasitology 122, S23-S38.

Crouch, A. C., Baxby, D., McCracken, C. M., Gaskell, R. M. and Bennett, M. (1995). Serological evidence for the reservoir hosts of cowpox virus in British wildlife. Epidemiology and Infection 115, 185-191.

Dare, O. K. and Forbes, M. R. (2008). Rates of development in male and female Wood Frogs and patterns of parasitism by lung nematodes. Parasitology 135, 385-393.
Dezfuli, B. S., Giari, L. and Poulin, R. (2001). Costs of intraspecific and interspecific host sharing in acanthocephalan cystacanths. Parasitology 122, 483-489.

Elftman, M. D., Norbury, C. C., Bonneau, R. H. and Truckenmillar, M. E. (2007). Corticosterone impairs dendritic cell maturation and function. Immunology 122, 279-290.

Elton, C. (1931). The study of epidemic diseases among wild animals. Fournal of Hygiene 31, 435-456.

Euzeby, J. (1982). From Echinococcus-Taenia biology in carnivore to man hydatidosis etiology and epidemiology. Revue De Medecine Veterinaire 133, 83-94.

Ferrari, N., Cattadori, I. M., Nespereira, J., Rizzoli, A. and Hudson, P. J. (2004). The role of host sex in parasite dynamics: field experiments on the yellow-necked mouse Apodemus flavicollis. Ecology Letters 7, 88-94.

Ferrari, N., Rosa, R., Pugliesa, A. and Hudson, P. J. (2007). The role of sex in parasite dynamics: Model simulations on transmission of Heligmosomoides polygyrus in populations of yellow-necked mice, Apodemus flavicollis. International Fournal for Parasitology 37, 341-349.

Galvani, A. P. and May, R. M. (2005). Epidemiology - dimensions of superspreading. Nature, London 438, 293-295.

Goater, C. P. (1992). Experimental population dynamics of Rhabdias bufonis (Nematoda) in toads (Bufo bufo) - density-dependence in the primary infection. Parasitology 104, 179-187.

Graham, A. L. (2008). Ecological rules governing helminth-microparasite co-infection. Proceedings of the National Academy of Sciences, USA 105, 566-570.

Gregory, R. D. (1991). Parasite epidemiology and host population growth - Heligmosomoides polygyrus (Nematoda) in enclosed wood mouse populations. Fournal of Animal Ecology 60, 805-821.

Gregory, R. D., Keymer, A. E. and Clarke, J. R. (1990). Genetics, sex and exposure - the ecology of Heligmosomoides poylgyrus (Nematoda) in the wood mouse. Fournal of Animal Ecology 59, 363-378.

Gregory, R. D., Montgomery, S. S. J. and Montgomery, W. I. (1992). Population biology of Heligmosomoides polygyrus (Nematoda) in the wood mouse. Fournal of Animal Ecology 61, 749-757.

Harder, A., Wunderlich, F. and Marinovski, P. (1992). Effects of testosterone on Heterakis spumosa infections in mice. Parasitology 105, 335-342.

Harvey, S. C., Paterson, S. and Viney, M. E. (1999). Heterogeneity in the distribution of Strongyloides ratti infective stages among the faecal pellets of rats. Parasitology 119, 227-235.

Hudson, P. J. and Dobson, A. P. (1995). Macroparasites: observed patterns. In Ecology of Infectious Diseases in Natural Population (ed. Grenfell, B. T. and Dobson, A. P.), pp. 144-176. Cambridge University Press, Cambridge, UK.

Janeway, C. A. and Travers, P. (1996). Immunobiology: The Immune System in Health and Disease. 2nd Edn. Garland Publishing, New York, NY, USA.

Keeling, M. J., Woolhouse, M. E. J., Shaw, D. J., Matthews, L., Chase-Topping, M., Haydon, D. T., Cornell, S. J., Kappey, J., Wilesmith, J. and Grenfell, B. T. (2001). Dynamics of the 2001 UK foot 
and mouth epidemic: Stochastic dispersal in a

heterogeneous landscape. Science 294, 813-817.

Keymer, A. E. and Hiorns, R. W. (1986).

Heligmosomoides polygyrus (Nematoda) - the dynamics of primary and repeated infection in outbred mice.

Proceedings of the Royal Society of London, B 229, 47-67.

Keymer, A. E. and Slater, A. F. G. (1987). Helminth fecundity - density dependence or statistical illusion. Parasitology Today 3, 56-58.

Keymer, A. E. and Tarlton, A. B. (1991). The population dynamics of acquired immunity to Heligmosomoides polygyrus in the laboratory mouse - strain, diet and exposure. Parasitology 103, 121-126.

Lello, J., Boag, B., Fenton, A., Stevenson, I. R. and Hudson, P. J. (2004). Competition and mutualism among the gut helminths of a mammalian host. Nature, London 428, 840-844.

Liz, J. S., Anderes, L., Sumner, J. W., Massung, R. F., Gern, L., Rutti, B. and Brossard, M. (2000). PCR detection of granulocytic ehrlichiae in Ixodes ricinus ticks and wild small mammals in western Switzerland. Fournal of Clinical Microbiology 38, 1002-1007.

Luong, L. T., Grear, D. A. and Hudson, P. J. (2009). Male hosts are responsible for the transmission of a trophically transmitted parasite, Pterygodermatites peromysci, to the intermediate host in the absence of sex-biased infection. International Fournal for Parasitology 39, 1263-1268.

Marcogliese, D. J. (1997). Fecundity of sealworm (Pseudoterranova decipiens) infecting grey seals (Halichoerus grypus) in the Gulf of St. Lawrence, Canada: Lack of density-dependent effects. International Fournal for Parasitology 27, 1401-1409.

Matthews, L., McKendrick, I. J., Ternent, H., Gunn, G. J., Synge, B. and Woolhouse, M. E. J. (2006). Super-shedding cattle and the transmission dynamics of Escherichia coli O157. Epidemiology and Infection 134, 131-142.

McClelland, G. (1980). Phocanema decipiens - growth, reproduction, and survival in seals. Experimental Parasitology 49, 175-187.

Michel, J. F. (1963). Phenomena of host resistance and course of infection of Ostertagia osteragi in calves. Parasitology 53, 63-84.

Montes, M., Sanchez, C., Verdonck, K., Lake, J. E., Gonzalez, E., et al. (2009). Regulatory T cell expansion in HTLV-1 and strongyloidiasis co-infection is associated with reduced IL-5 responses to Strongyloides stercoralis antigen. PLoS Neglected Tropical Diseases 3, e456. doi:10.1371/journal.pntd.0000456.

Noble, G. A. (1961). Stress and parasitism. I. A preliminary investigation of effects of stress on ground squirrels and their parasites. Experimental Parasitology 11, 63-67.

Noble, G. A. (1962). Stress and parasitism. II. Effect of crowding and fighting among ground squirrels on their coccidia and trichomonads. Experimental Parasitology 12, 368-371.

Noble, G. A. (1966). Stress and parasitism. III. Reduced night temperature and effect on pinworms of ground squirrels. Experimental Parasitology 18, 61-62.

O'sullivan, H. M., Smal, C. M. and Fairley, J. S. (1984). A study of parasite infestations in populations of small rodents (Apodemus sylvaticus and Clethrionomys glareolus) on Ross Island, Killarney. Fournal of Life Sciences Royal Dublin Society 5, 29-42.

Paterson, S. and Barber, R. (2007). Experimental evolution of parasite life-history traits in Strongyloides ratti (Nematoda). Proceedings of the Royal Society London, B 274, 1467-1474.

Paterson, S. and Viney, M. E. (2002). Host immune responses are necessary for density dependence in nematode infections. Parasitology 125, 283-292.

Perkins, S. E., Cagnacci, F., Stradiotto, A., Arnoldi, D. and Hudons, P. J. (2009). Comparison of social networks derived from ecological data: implications for inferring infectious disease dynamics. Fournal of Animal Ecology 78, 1015-1022.

Perkins, S. E., Cattadori, I. M., Tagliapietra, V., Rizzoli, A. P. and Hudson, P. J. (2003). Empirical evidence for key hosts in persistence of a tick-borne disease. International Fournal for Parasitology 33, 909-917.

Perkins, S. E., Cattadori, I. M., Tagliapietra, V., Rizzoli, A. P. and Hudson, P. J. (2006). Localized deer absence leads to tick amplification. Ecology 87, 1981-1986.

Poulin, R. (1996). Helminth growth in vertebrate hosts: Does host sex matter? International Fournal for Parasitology 26, 1311-1315.

Quinnell, R. J. (1992). The population dynamics of Heligmosomoides polygyrus in an enclosure population of wood mice. Fournal of Animal Ecology 61, 669-679.

Ractliffe, L. H. and LeJambre, L. F. (1971). Increase of rate of egg production with growth in some intestinal nematodes of sheep and horses. International Fournal for Parasitology 1, 153-156.

Rasband, W. (2007). Imagef. U.S. National Institutes of Health, Bethesda, MD, USA.

Rizzoli, A., Neteler, M., Rosa, R., Versini, W., Cristofolini, A., Bregoli, M., Buckley, A. and Gould, E. A. (2007). Early detection of tick-borne encephalitis virus spatial distribution and activity in the province of Trento, northern Italy. Geospatial Health 1, 169-176.

Scott, M. E. (1987). Regulation of mouse colony abundance by Heligmosomoides polygyrus. Parasitology 95, 111-124.

Scott, M. E. (1990). An experimental and theoretical study of the dynamics of mouse nematode (Heligmosomoides polygyrus) interaction. Parasitology 101, 75-92.

Shaw, D. J. and Dobson, A. P. (1995). Patterns of macroparasite abundance and aggregation in wildlife populations: A quantitative review. Parasitology 111, S111-S133.

Smith, J. W. (1988). An electronic method for estimating the vaginal and uterine egg content of nematodes, with special reference to ascaridoids. Canadian Fournal of Zoology 66, 2253-2254.

Smith, D. L., McKenzie, F. E., Snow, R. W. and Hay, S. I. (2007). Revisiting the basic reproductive number for malaria and its implications for malaria control. PLoS Biology 5, e42. doi:10.1371/journal.pbio.0050042.

Stear, M. J., Bairden, K., Duncan, J. L., Holmes, P. H., McKellar, Q. A., Park, M., Strain, S., Murray, M., Bishop, S. C. and Gettinby, G. (1997). How hosts control worms. Nature 389, 27. 
Stear, M. J. and Bishop, S. C. (1999). The curvilinear relationship between worm length and fecundity of Teladorsagia circumcincta. International Fournal for Parasitology 29, 777-780.

Stear, M. J., Bishop, S. C., Doligalska, M., Duncan, J. L., Holmes, P. H., Irvine, J., McCririe, L., McKellar, Q. A., Sinski, E. and Murray, M. (1995). Regulation of egg production, worm burden, worm length and worm fecundity by host responses in sheep infected with Ostertagia circumcincta. Parasite Immunology 17, 643-652.

Stein, M. and Schleifer, S. J. (1985). Frontiers of stress research: stress and immunity. In Stress in Helath and Disease (ed. Zales, M.), pp. 97-114. Brunner/Mazel, New York, USA.

Swanson, J. A., Falvo, R. and Bone, L. W. (1984). Nippostrongylus brasiliensis - effects of testosterone on reproduction and establishment. International fournal for Parasitology 14, 241-247.

Szalai, A. J. and Dick, T. A. (1989). Differences in numbers and inequalities in mass and fecundity during the egg producing period for raphid Ascaris acus (Nematoda, Anisakidae). Parasitology 98, 489-495.

Tagliapietra, V., Rosa, R., Hauffe, H. C., Laakkonen, J., Voutilainen, L., Vapalahti, O., Vaheri, A., Henttonen, H. and Rizzoli, A. (2009). Spatial and temporal dynamics of lymphocytic choriomeningitis virus in wild rodents, Northern Italy. Emerging Infectious Diseases 15, 1019-1025.

Tanguay, G. V. and Scott, M. E. (1992). Factors generating aggregation of Heligmosomoides polygyrus (Nematoda) in laboratory mice. Parasitology 104, 519-529.

Telfer, S., Bennett, M., Carslake, D., Helyar, S. and Begon, M. (2007). The dynamics of murid gammaherpesvirus 4 within wild, sympatric populations of bank voles and wood mice. Fournal of Wildlife Diseases 43, 32-39.

Tompkins, D. and Hudson, P. J. (1999). Regulation of nematode fecundity in the ring-necked pheasant (Phasianus colchicus): not just density dependence. Parasitology 118, 417-423.

Viney, M. (2002). How do host immune responses affect nematode infections? Trends in Parasitology 18, 63-66.

Wilson, K., Bjornstad, O. N., Dobson, A. P., Merler, S., Poglayen, G., Randolf, S. E., Read, A. F. and Skorping, A. (2001). Heterogeneities in macroparasite infections: patterns and processes. In The Ecology of Wildlife Disease (ed. Hudson, P. J., Rizzoli, A., Grenfell, B. T., Heesterbeek, H. and Dobson, A. P.), pp. 6-44. Oxford University Press, Oxford, UK.

Woolhouse, M. E. J., Dye, C., Etard, J. F., Smith, T., Charlwood, J. D., Garnett, G. P., Hagan, P., Hii, J. L. K., Ndhlovu, P. D., Quinnell, R. J., Watts, C. H., Chandiwana, S. K. and Anderson, R. M. (1997). Heterogeneities in the transmission of infectious agents: Implications for the design of control programs. Proceedings of the National Academy of Sciences, USA 94, 338-342.

Woolhouse, M. E. J., Watts, C. H. and Chandiwana, S. K. (1991). Heterogeneities in transmission rates and the epidemiology of Schistosome infection. Proceedings of the Royal Society of London. B 245, 109-114.

Zuk, M. (1990). Reproductive strategies and disease susceptibility: an evolutionary viewpoint. Parasitology Today 6, 231-233.

Zuk, M. and McKean, K. A. (1996). Sex differences in parasite infections: Patterns and processes. International Fournal for Parasitology 26, 1009-1023. 